\title{
The mechanical properties and modeling of a sintered, hollow sphere, steel foam
}

\author{
ST Szyniszewski ${ }^{a 1}$, BH Smith ${ }^{b}$, JF Hajjar $^{\mathrm{c}}$, BW Schafer ${ }^{\mathrm{d}}$, SR Arwade \\ ${ }^{a}$ Dept. of Civil and Env. Eng., University of Surrey, Guildford, GU2 7XH \\ ${ }^{b}$ Dept. of Civil and Env. Eng., University of Massachusetts, Amherst, MA 01003 \\ ${ }^{c}$ Dept. of Civil and Env. Eng., Northeastern University, Boston, MA 02115 \\ ${ }^{d}$ Dept. of Civil Eng., Johns Hopkins University, Baltimore, MD 21218
}

\begin{abstract}
This paper characterizes mechanical properties of hollow sphere (HS) steel foam, and applies calibrated Deshpande-Fleck plasticity to mechanical simulations of steel foam components. Foamed steel, steel with internal voids, provides enhanced bending rigidity, exceptional energy dissipation, and the potential to mitigate local instability. The experimental characterization of the hollow sphere foam encompasses compressive yield stress and densification strain, compressive plastic Poisson's ratio, compressive unloading modulus, as well as tensile elastic modulus, tensile unloading modulus, tensile yield stress, tensile fracture strain, and shear yield stress and fracture strain. Since HS steel foam is compressible under triaxial pressure, Deshpande-Fleck plasticity of compressible metals was calibrated and employed in simulations. Plastic Poisson's ratio, measured in a uniaxial test, is an important metric of foam compressibility, and it affects the response of the foam to multi-axial loadings significantly. This work is part of a larger effort to help develop steel foam as a material with relevance to civil engineering applications.
\end{abstract}

Keywords: steel foam, metal foam, compression, tension, shear, experimental characterization, foam plasticity, foam fracture, foam failure, plastic Poisson's ratio

\footnotetext{
${ }^{1}$ Corresponding author at: 01 AA 03, Thomas Telford Building, Guildford, Surry, GU2 7XH, UK. Tel.: +44 148368 3390. E-mail address: s.szyniszewski@surrey.ac.uk|s.szyniszewski@gmail.com
} 


\section{Introduction and background}

Developments in the manufacturing, processing, and applications of novel metallic foams offer a promise for a significant technological advancement of metallic materials. Steel foam is porous steel, with intentionally introduced voids varying in size from nano- to millimeters. Porosity of steel foam can be controlled, and since it affects mechanical properties of the material, Young's modulus and yield stress can be tuned for specific needs by adjusting the foam porosity [2]. Mutlu et al. [3] reported successful manufacturing of $\mathrm{Cr}-\mathrm{Si}-\mathrm{Ni}-\mathrm{Mo}$ based steel foams, and $\mathrm{Hsu}$ et al. produced $\mathrm{Ti}-7.5 \mathrm{Mo}$ alloy metallic foam [4]. These recent developments indicate that increasingly exotic metallic materials are employed in metallic foams.

Metallic foams, when properly designed, provide lighter and stiffer elements than solid steel components [2]. Also, metallic foams are renowned for their compressibility of 0.9 engineering strain or more [5], giving them extraordinary energy dissipation capacity [2], which is instrumental in arresting extreme dynamic events because it dissipates the kinetic energy. Once the kinetic energy is removed completely, the system reaches a stable state [6,7]. Energy dissipation capacity has been experimentally observed in metallic foams even under high strain rates [8].

Metal foams enable components with higher buckling resistance and lower weight in comparison to traditional solid steel structures [9]. Sandwich panels, with a metallic foam core, increase buckling resistance even further [10]. "An important structural advantage of metallic foams that has not been demonstrated to date is the conversion of limit states from unstable buckling modes with little or no energy dissipation to stable modes with crashing and/or post-buckling" [1]. 
Sandwich panels, with a metallic foam core, have bending stiffness to weight ratio an order of magnitude greater than a solid plate of the same weight [11]. A parking garage metallic foam floor slab was manufactured, and full-scale load tests carried out successfully. The use of the metal mesh reinforced panels reduced the weight of the floors by $75 \%$ in comparison to conventional reinforced concrete decks [12]. Lefebvren et al. [13], Banhart and Seeliger [11] and Smith et al. [1] provide an extensive overview of current industrial applications of metallic foams.

Multi-physics properties of metallic foams such as: lower thermal conductivity [14], vibration attenuation [15], sound absorption [16], fluid flow through open cells [15], fire resistance [17], electromagnetic [18] and radiation [19] shielding give metallic foams an advantage over conventional materials with comparable structural characteristics. There is great potential for multi-functional applications, though such designs require more sophisticated analysis methodologies.

Metal foams differ from solid steel significantly, and warrant novel modeling approaches. Steel foam is compressible after yield [20], unlike solid steel, which exhibits only shear deformations and is incompressible in the plastic regime. Also, foams often fracture under tensile strains noticeably lower than crushing and compaction strains [21], thus warranting a tailored failure criterion.

This research not only characterizes novel steel foams, but also investigates calibration of plasticity formulations suitable for porous metals, such as Deshpande-Fleck (D-F) plasticity [20]. Also, failure criteria available in the literature [21,22] are evaluated against experimental results, and alternative approaches to failure modeling are proposed. This work is specifically focused on steel foam produced by the sintering of metal hollow spheres, but the principles may be applicable to a much greater variety of metal foams.

\section{Materials and methods}

Fifty hollow sphere (HS) steel foam bars of approximate relative density 0.15 were acquired from the Fraunhofer Institute for Advanced Materials (IFAM) in Dresden, 
Germany. Each bar measures approximately $250 \mathrm{~mm} \times 52 \mathrm{~mm} \times 55 \mathrm{~mm}$ and is composed of a mild steel of between $0.3 \%$ and $0.5 \%$ carbon. Measurement of the geometry of 50 spheres under an optical microscope at five times magnification yielded mean sphere diameter of $1.86 \mathrm{~mm}$ and mean sphere thickness of $0.08 \mathrm{~mm}$.

Test specimen geometry and loading characteristics for compression, tension, and shear tests were selected to follow guidance provided by international standards as closely as possible while accommodating the constraints imposed by the available testing equipment and the characteristics of the Fraunhofer HS foam, including the challenges presented by machining it. Details of the specimens and test setup used in each of the three tests is described in the following sections, and shown photographically in figure 1. The HS foam, which is formed by sintering the hollow steel spheres together, contains relatively weak bonds between the spheres, and we found that lathes, mills, and other rotational tools were ineffective at smoothly removing material, instead removing entire spheres or clusters of spheres and leaving highly irregular surfaces. However, cutting the material with a band saw with a fine-tooth blade was effective, though it was difficult to form complicated geometries with this technique.
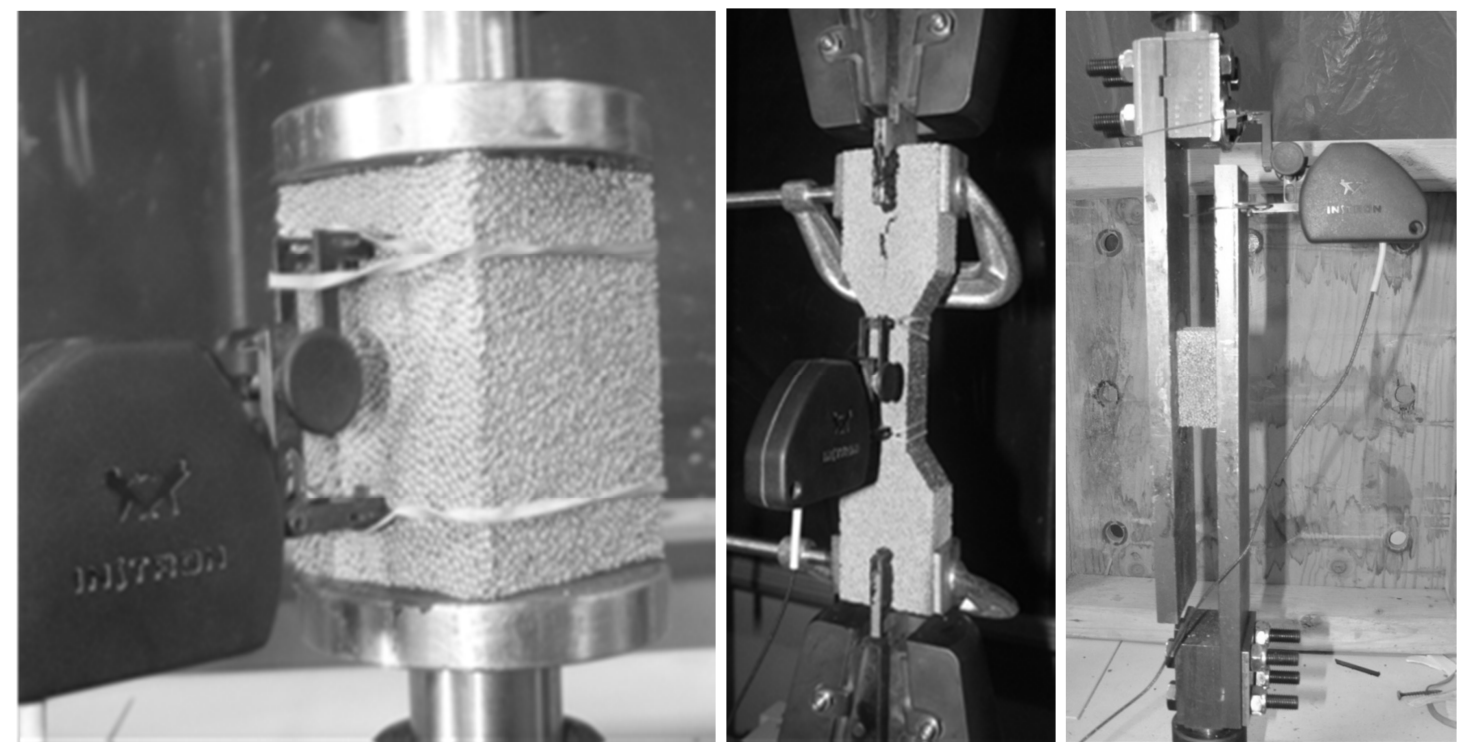

Figure 1: From left to right: (a) Compression test with longitudinal extensometer; (b) Mounted tension specimen with longitudinal extensometer; (c) Mounted shear specimen with longitudinal extensometer in the upper right. 


\subsection{Compression specimens and testing protocol}

For compression, draft standard ISO/DIS 13314 "Compression Test for Porous and Cellular Metals" [23], suggests that rectilinear prismatic specimens should have a square cross-section and have a height to width ratio of between 1.5 and 2.0. We selected a height-to-width ratio of 1.45 to $1.55(80 \mathrm{~mm} \times 52 \mathrm{~mm} \times 55 \mathrm{~mm})$ so that three test specimens could be machined from each $250 \mathrm{~mm}$ x $52 \mathrm{~mm}$ x $55 \mathrm{~mm}$ bar. Specimens with dimensions $25 \mathrm{~mm} \times 25 \mathrm{~mm} \times 55 \mathrm{~mm}$ were machined for tests designed to capture the large strain densification behavior of the material.

The compression specimens were tested in a screw driven Instron 3369 material testing machine between flat stainless steel platens that were lubricated with a standard heavy axle grease at applied strain rates between $10^{-3.7} \mathrm{~s}^{-1}$ and $10^{-4.0} \mathrm{~s}^{-1}$. This range of strain rates ensures slower load application than the minimum strain rate of $10^{-3.0} \mathrm{~s}^{-1}$ defined in the ISO/DIS standard, and was selected to allow manual measurement of the transverse strain during compression testing. Transverse strain is required for calculation of the material Poisson's ratio, a critical calibration parameter for the constitutive model described later in this paper.

Table 1 defines the parameters of the three compression test types conducted during this study. These three tests were designed to allow evaluation of the elastic modulus, yield stress, Poisson's ratio, and densification strain. The test specimen in its loading fixture is shown in Fig. 1.

Table 1: Compression test characteristics

\begin{tabular}{|c|c|c|c|c|}
\hline Measurement & Loading type & Strain rate $\mathrm{s}^{-1}$ & Strain acquisition & Specimen size \\
\hline $\begin{array}{l}\text { Densification } \\
\text { strain }\end{array}$ & $\begin{array}{l}\text { Monotonic } \\
\text { compression }\end{array}$ & $10^{-3.7}$ & $\begin{array}{l}\text { Crosshead } \\
\text { displacement }\end{array}$ & $\begin{array}{l}55 \mathrm{~mm} \times 25 \mathrm{~mm} \\
\times 25 \mathrm{~mm}\end{array}$ \\
\hline Poisson's ratio & $\begin{array}{l}\text { Monotonic } \\
\text { compression }\end{array}$ & $10^{-3.7}$ & $\begin{array}{l}\text { Transverse } \\
\text { extensometer }\end{array}$ & $\begin{array}{l}80 \mathrm{~mm} \times 52 \mathrm{~mm} \\
\times 55 \mathrm{~mm}\end{array}$ \\
\hline $\begin{array}{l}\text { Elastic modulus / } \\
\text { yield stress }\end{array}$ & $\begin{array}{l}\text { Compression, } \\
\text { unloadings spaced at } \\
0.5 \% \text { to } 1.0 \% \text { strain }\end{array}$ & $\begin{array}{l}10^{-3.7} \text { loading } \\
10^{-4.0} \text { unloading }\end{array}$ & $\begin{array}{l}\text { Loading direction } \\
\text { extensometer }\end{array}$ & $\begin{array}{l}80 \mathrm{~mm} \times 52 \mathrm{~mm} \\
\times 55 \mathrm{~mm}\end{array}$ \\
\hline
\end{tabular}




\subsection{Tension specimens and testing protocol}

ASTM E8, "Tension testing of metallic materials", provides the most relevant guidance for the testing of steel foam in tension due to the lack of a published standard for tension testing of metal foams. The significantly different characteristics of steel foam as compared to solid metals, however, necessitates several modifications to the specimens and testing protocols defined in that standard.

Reduced workability of the material prevented us from machining smooth rounded fillets, and the specimen geometry was therefore adopted and readily machined by cutting on a band saw. The overall specimen dimensions are consistent with those specified in the ASTM standard. In place of the wedge grips specified in the standard, which would crush the grip section of the specimen, we developed a gripping mechanism that used a solid steel plate epoxied into a notch in the specimen to transfer load between the wedge grips of the Instron machine and the steel foam sample (figure 1(b)). An initial test using this configuration resulted in the fracture emanating from the corners of the notch but the addition of a clamping force (as shown in the figure) provided sufficient confinement to drive fracture into the test section. Tests were run at a strain rate of $10^{-2.3} \mathrm{~s}^{-1}$ based on the recommended strain rate for compression testing of steel foams. Three samples were tested under this protocol with an extensometer used to collect strain data in the loading direction, and a fourth test was conducted with an added unloading stage prior to the onset of nonlinearity to evaluate the elastic modulus in tension.

\subsection{Shear specimens and testing protocol}

Shear testing was performed following the ISO 1922 [25] standard for testing of cellular plastics, which involves attaching a thin rectangular sample to two rigid platens, and then pulling one platen in a direction parallel to the platen's face. The ISO 1922 testing standard calls for specimens of size $25 \mathrm{~mm}$ by $50 \mathrm{~mm}$ by $250 \mathrm{~mm}$. After multiple attempts at performing such tests, however, it was found to not be possible to machine a flat enough surface on the hollow spheres foam so that the entire surface would end up laminated by the epoxy, as only limited quantities of epoxy strong enough to hold the material was available. Therefore, the ISO 1922 standard dimensions were reduced; the 
$25 \mathrm{~mm}$ thickness was kept, but the depth was reduced from $50 \mathrm{~mm}$ to $25 \mathrm{~mm}$, and then height from $250 \mathrm{~mm}$ to $55 \mathrm{~mm}$. Three such tests were performed. Devcon Plastic Welder epoxy was used for attaching the samples to the platens for these shear tests. The ISO 1922 standard was followed precisely for the remainder of the testing procedure, and no further deviations were necessitated by the use of steel foam in the tests. Platens for use in this test were custom manufactured, as none previously existed that would serve the purpose. In order to measure strains, an extensometer was attached between the lower platen and the upper. A photograph of the final setup is shown in figure 1(c).

\section{Experimental results}

\subsection{Densification}

Densification is the process by which, under large strain compressive loading, the cells of a foam begin to collapse, resulting in contact between opposite faces of the cells. This contact results in rapid stiffening of the material. The increased stress transmission may either be desirable or undesirable depending on the design context, but either way must be accounted for during the design process.

Three replications of the densification compression test were performed resulting in the stress-strain curves shown in figure 2. Densification begins at a strain of approximately 0.65. No established definition exists for the onset of densification, and we have adopted the following definition: Let $E_{t, 0.05}(\varepsilon)$ be the tangent modulus of the material determined by performing a linear regression on the stress strain curve over the range $[\varepsilon-0.05$,

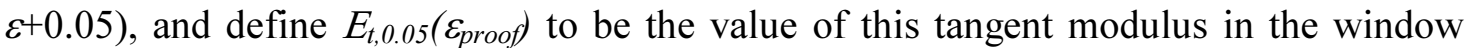
immediately following the 0.01 proof stress (essentially a 0.01 offset version of the yield stress). We define the densification strain to be:

$$
\varepsilon_{d}=\min \left\{\varepsilon: E_{t, 0.05}(\varepsilon)>E_{t, 0.05}\left(\varepsilon_{\text {proof }}\right)\right\}
$$

meaning that densification is assumed to begin when the tangent modulus exceeds for the first time the post-yield tangent modulus. This definition gives an average densification 
strain for the three samples of $\varepsilon_{d}=0.65$ with a range of \pm 0.03 . The tests also revealed that the material exhibits a substantial hardening modulus between the yield and densification onset of approximately $25 \mathrm{MPa}$. The presence of such a hardening modulus should give pause to analysts who prefer to use an elastic-perfectly plastic material model. Despite the small value of the hardening modulus, the large strains that can be absorbed by the material mean that the stress level increases by a factor of two between yield and densification, a feature that a perfectly plastic model would fail to capture.

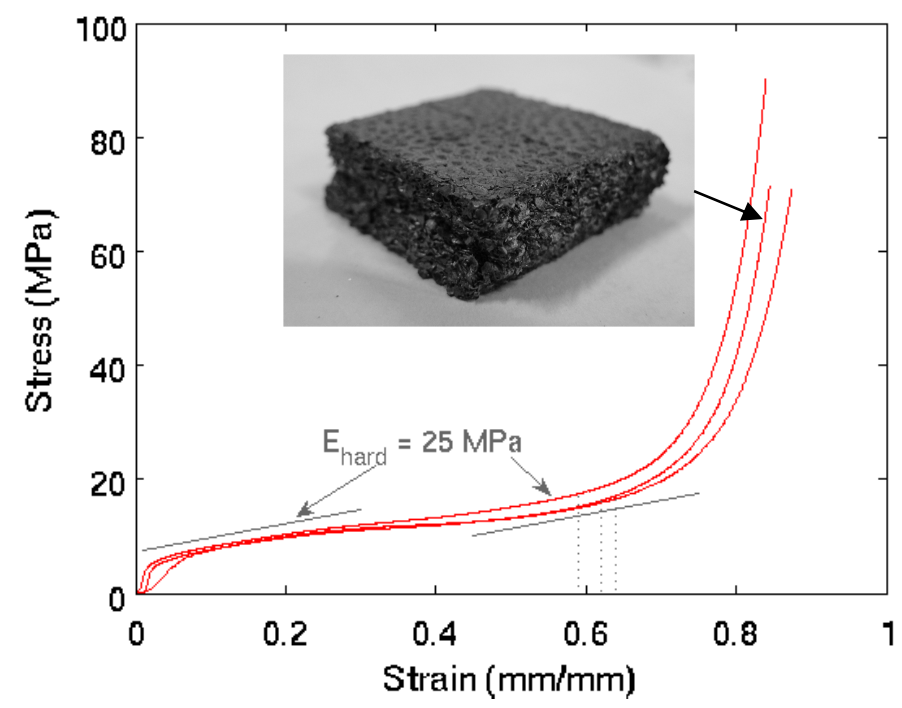

Figure 2: Stress strain curves for nominally identical hollow spheres steel foam samples tested in compression into the densification range, showing initial elastic loading, hardening plateau, and sharp increase in material stiffness after initiation of densification. Inset photograph shows a densification sample after testing to approximately 0.85 strain showing collapse of hollow spheres and small asymmetry in the transverse deformations.

Note that all strain values for this test are based upon measurements taken from the crosshead displacement of the Instron. Manual measurements and comparison with extensometer-acquired strain data collected in other tests indicates that the strain readings, while not accurate enough to estimate the initial elastic modulus, do provide accurate measurement in the post yield regime as the strains become large.

\subsection{Poisson's ratio}

For isotropic elastic continuum analysis, a single value of Poisson's ratio is sufficient to fully characterize the material response since standard plastic flow rules assume 
incompressibility, and a Poisson's ratio of 0.5 in the post-yield regime. Foams, however, undergo significant volume change even after yield, and a more complete understanding of the Poisson's ratio of steel foams is needed to allow mechanistically rigorous design approaches.

Three replications of the Poisson's ratio compression test, run with the extensometer mounted transverse to the loading direction, were performed and used to evaluate the evolution of the Poisson's ratio with applied compressive strain. To calculate the evolving value of the Poisson's ratio, we have assumed that the two transverse components of the engineering strain $\left(\varepsilon_{x}\right.$ and $\left.\varepsilon_{z}\right)$ are equal, and have calculated the average Poisson's ratio over increments of applied compressive strain $\varepsilon_{y}$ of length 0.005 . By this definition the Poisson's ratio is given as a function of position by:

$$
v\left(\varepsilon_{y}\right)=\left[\varepsilon_{x}\left(\varepsilon_{y}+0.005\right)-\varepsilon_{x}\left(\varepsilon_{y}\right)\right] \div 0.005
$$

where $\varepsilon_{x}\left(\varepsilon_{y}\right)$ represents the value of the transverse strain $\varepsilon_{x}$ evaluated at applied strain $\varepsilon_{y}$.

Figure 3 shows the complicated evolution of Poisson's ratio with increasing applied strain, with a fairly rapid increase from near zero to a peak value at an applied strain of approximately 0.4 which falls in the middle of the post-yield plateau observed in figure 2 . The test was terminated at about 0.6 engineering strain, because insufficient space was remaining in the test fixture to accommodate the transverse-mounted extensometer.

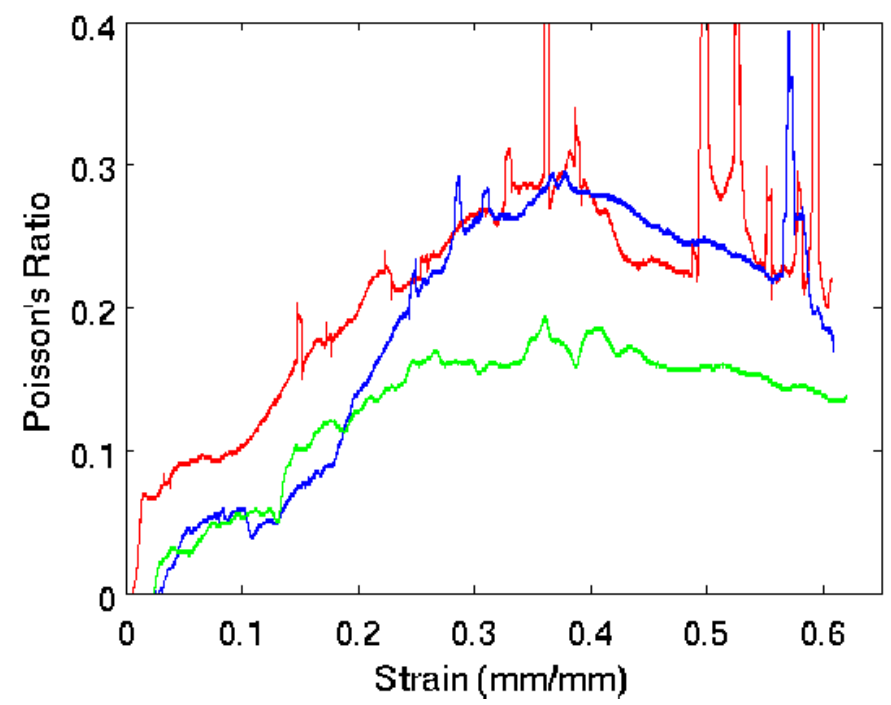


Figure 3: Instantaneous Poisson's ratio, calculated by dividing transverse engineering strain by longitudinal engineering strain over $0.5 \%$ longitudinal strain intervals.

The Poisson's ratio of $0.32-0.34$, which is derived based on elementary mechanics of cellular networks [5], may not apply for all foams. In the case of the HS foam tested here, the peak value of Poisson's ratio is in the mechanistically derived range, but over almost all of the range tested, the HS foam exhibits a Poisson's ratio much lower than 0.3. This finding is significant for the behavior of HS steel foam under multi-axial stress states and even under uniaxial loading where the level of confinement in the interior of the specimen would be much lower than predicted by $0.31-0.34$ Poisson's ratio values.

\subsection{Modulus through multiple unloadings}

Measuring elastic modulus accurately during initial loading is challenging due to imperfections in specimen preparation, test fixtures, and loading apparatus. To determine the elastic modulus of the HS foam, therefore, we conducted uniaxial compression tests with multiple unloading segments. By repeating the unloading at regular intervals during the course of the test we were also able to track any evolution in the modulus of the material. These tests, replicated six times, with an extensometer used to measure strain in the direction of applied load, were also used to measure the yield stress of the material.

Longitudinal strain $\varepsilon_{y}$ was recorded using both the extensometer and the crosshead displacement of the testing machine. Before strains of approximately 0.05 , the extensometer- and crosshead-based strains differed substantially, with the extensometer measuring lower strain values than the crosshead. After approximately 0.05 applied strain the two values were acceptably close to one another. This observation lends support to our use of crosshead displacement for measuring $\varepsilon_{y}$ during the densification and Poisson's ratio tests, when most of our attention was focused on large strain behavior. Because the extensometer was placed in the middle of the specimen, the fact that it records lower strain readings than the crosshead indicates significant deformation early in the loading history as the top and bottom surfaces of the specimen come into contact with 
the loading platens. This is to be expected because the limited workability of the material made it difficult to obtain flat and parallel loading faces to within tight tolerances.

We tested two sets of specimens of identical cross section $(52 \mathrm{~mm} \times 55 \mathrm{~mm})$, but different heights $(80 \mathrm{~mm}$ and $140 \mathrm{~mm})$. From the data shown in figure 4, we extracted a post-yield hardening modulus of $25 \mathrm{MPa}$, and found that the 0.002 offset yield stress averaged $\sigma_{y, 0.002}=3.2 \pm 0.6 \mathrm{MPa}$. Figure 6 shows substantial variation in the stress strain response of the material at strains lower than roughly 0.02 , but that at strains greater than 0.02 the variability decreases. The 0.002 offset yield stress captures this early variability, but in designing steel foam applications in which moderate to large deformations are to be expected the 0.002 offset stress may overestimate the practical variability of the material properties.
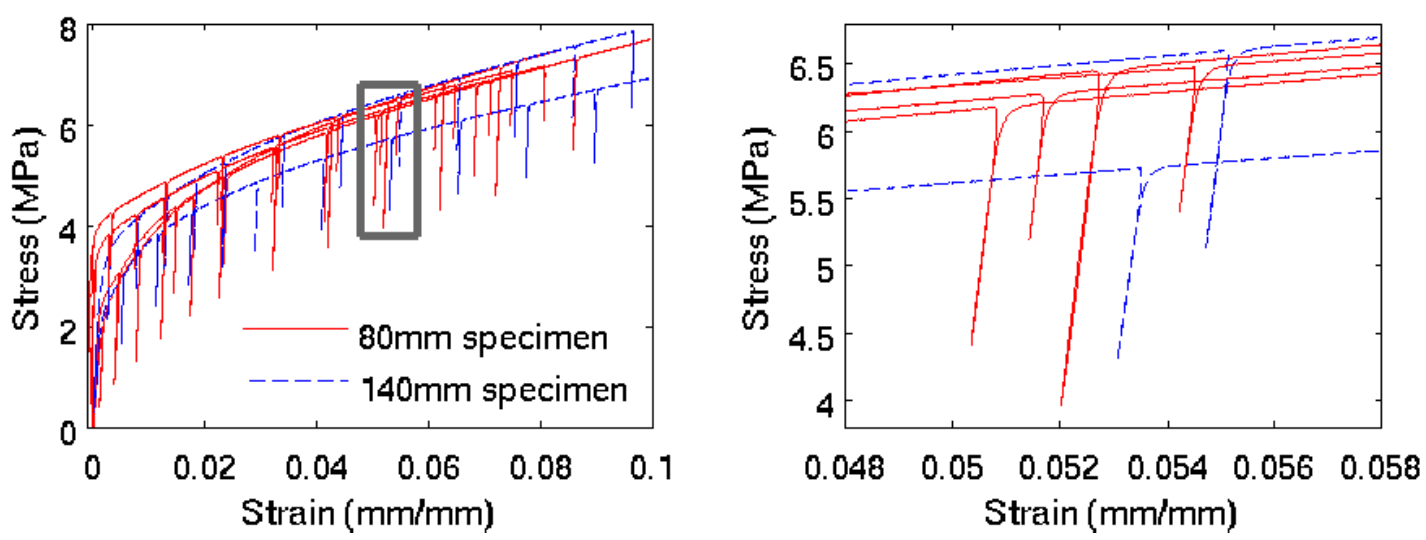

Figure 4: Stress-strain curves for multiple unloading tests. Left: full test. Right: zoomed plot.

To provide another estimate of yield stress that would capture the reduced variability present in the post-elastic regime, we adopted the practice of the ISO/DIS 13314 [23] standard which suggest use of an 0.01 proof stress, which is defined simply as the stress value at an applied strain of 0.01 . For the HS foam tested here, the 0.01 proof stress averaged $\sigma_{p, 0.01}=4.0 \pm 0.3 \mathrm{MPa}$. The choice of yield stress is a particularly important consideration because of the frequency with which bi- or tri-linear material models are used in practical analysis, and we suggest that $\sigma_{\mathrm{p}, 0.01}=4.0 \pm 0.3 \mathrm{MPa}$ is a better choice than $\sigma_{\mathrm{y}, 0.02}=3.2 \pm 0.6 \mathrm{MPa}$, and is more reflective of the actual material behavior. 
This series of tests also provides the best measurement of the elastic modulus of the HS steel foam. The elastic modulus was estimated by performing a least square linear regression on each of the unloading episodes shown in figure 4 . The results indicated a rather large amount of uncertainty early in the loading history - the first two unloadings occur in the initial elastic range — but become quite constant after an applied strain of $\varepsilon_{y}=$ 0.02. Even after the modulus values in each test become essentially constant, there remains substantial inter-specimen variability with $\mathrm{E}_{\mathrm{y}}=3150 \pm 250 \mathrm{MPa}$. The test results show no strong evidence for evolution of the elastic modulus during deformation, at least up to an applied strain of 0.1 , indicating that although the material is well past yield at that point, no substantial damage has yet accumulated at the microstructural level. The highly variable moduli measured prior to $\varepsilon_{y}=0.02$ are, in our judgment, due to initial imperfections in the test specimens, and should not be regarded as characteristics of the material.

It is difficult to compare the elastic modulus we measured to other published values, as few published HS foams have been above a relative density of 0.08 , and the Fraunhofer HS foam we tested had relative density of approximately 0.15 . However, Friedl et al. [26] measured the elastic modulus of their $\rho=0.08$ steel foam as being $640 \mathrm{MPa}$, compared to the $3150 \mathrm{MPa}$ measured for this $\rho=0.15$ foam. Other material properties, including yield stress (3.4 MPa) and hardening modulus (30 MPa), were all within $10 \%$ of the values reported by Friedl et al., but these comparisons are hard to attach meaning to without more knowledge of the base material properties. Note that Young's modulus and yield stress obtained are within the bounds set by the Gibson and Ashby [2] models.

\subsection{Tension tests}

Three replications of a uniaxial tension test were conducted to evaluate the behavior of the HS foam in tension, which have not been reported previously in the literature. The results, summarized in the stress strain curves of figure 5 , indicate poor tensile ductility for this material, with fracture strains $\varepsilon_{f}=0.019 \pm 0.007$, and a high level of uncertainty associated with the fracture strain. The peak tensile stresses, $\sigma_{f}=4.9 \pm 0.9 \mathrm{MPa}$ vary 
noticeably, although the strength in tension is comparable to the stress in compression at equal strain levels. In an additional test, which is not shown in the figure, several periods of unloading were included to evaluate the material elastic modulus in tension. No significant difference was found between the compressive and tensile moduli.

In two of the three replications two dominant cracks formed, originating from opposite sides of the specimen, while in the other replication a single dominant crack formed. In two of the three replications the dominant $\operatorname{crack}(\mathrm{s})$ formed well away from the transition from the grip to test sections leading us to conclude that the specimen design, despite its small deviations from the most relevant ASTM [24] standard, is appropriate for characterizing the tensile material properties of this HS steel foam. The shape of the stress strain curve in all three tests was similar up to the point of peak stress, the point at which a dominant crack becomes manifest in the specimen. In all three cases a distinct yield point and a small amount of inelastic deformation was observed, meaning that the material does not act as a completely brittle solid in tension. The nearly immediate drop to zero stress level in replication 3 was recorded because the dominant crack appeared at the location of one of the extensometer blades, meaning that additional strain ceased to be recorded. The crosshead-based stress strain curves, which are not shown here, indicate that the post-peak behavior was similar for all three tests.

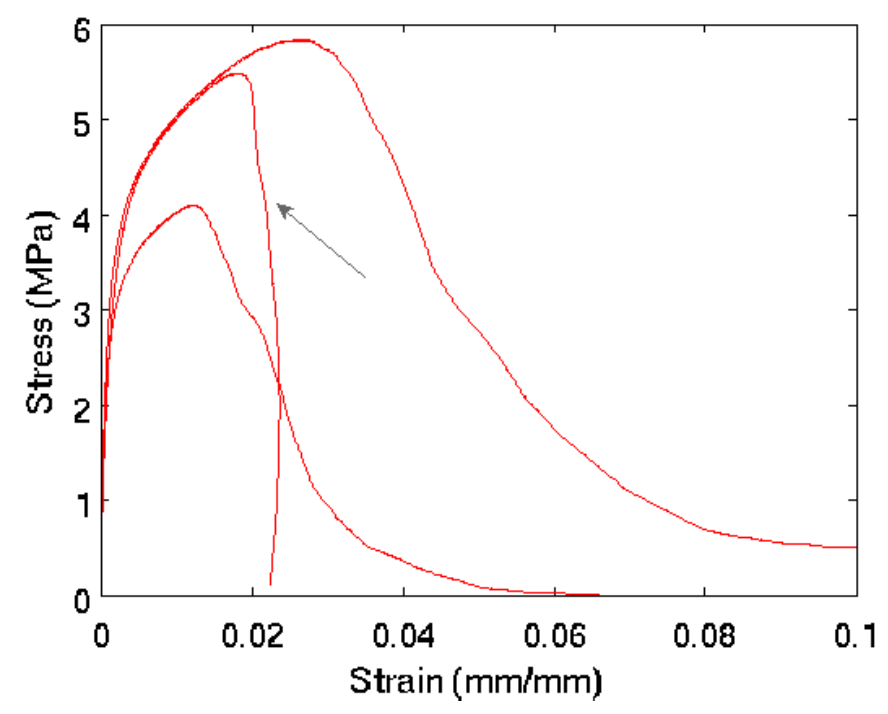



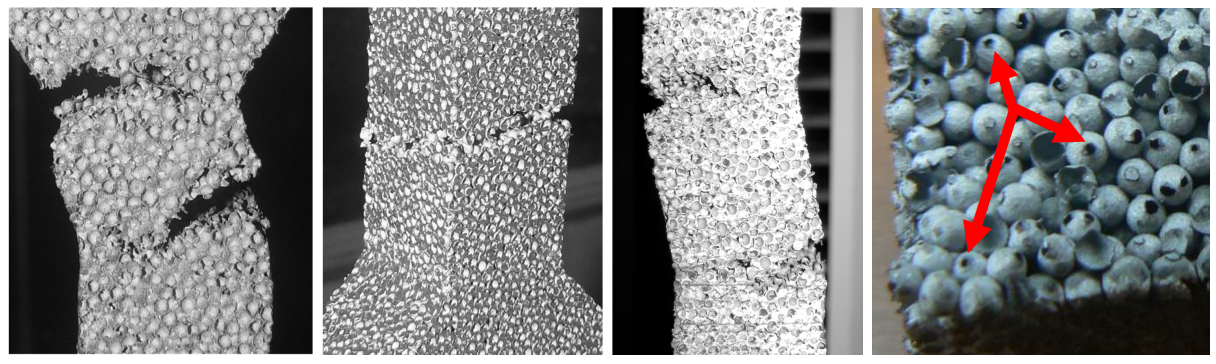

Figure 5: Above: Stress-strain curves of tension tests. Note that the test indicated by the arrow shows a more sudden drop in stress due to the crack having formed nearly directly underneath one of the blades of the extensometer. Below: The fracture surfaces for each test, including one macro photograph (far right).

Fracture occurred by failure of the individual hollow spheres at the points where they were sintered to adjoining sphere (figure 5, bottom far right). The fracture is located in the spheres because the connections between spheres, where the material thickens substantially (figure 6), are stronger than the hollow sphere shells themselves, and indicates that if greater tensile strength - and possibly ductility-is desired from the material, thicker spheres should be used. If the diameter of the spheres were increased this change could be made without affecting the overall relative density of the material.

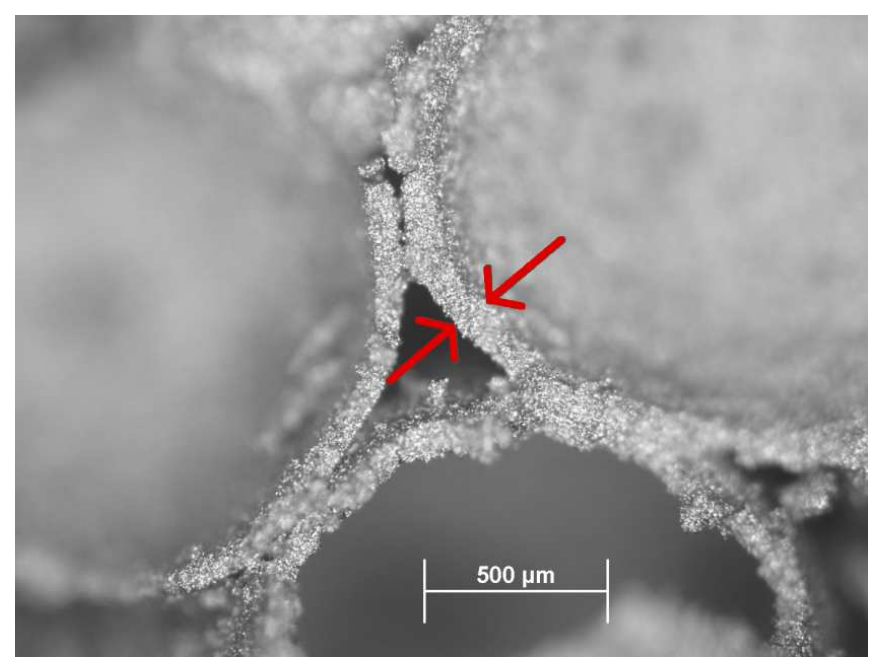

Figure 6: Sphere wall thickness microscopy measurement. 


\subsection{Shear tests}

Shear experiments showed remarkable consistency, with shear modulus and ultimate stress varying by less than $\pm 10 \%$ (see figure 7 and table 2 ). Limited ductility is evident because the material yields before it reaches its ultimate strength. There are also two distinct slopes in the post-yield behavior. The second, smaller slope, beginning at about 0.07 shear strain, is likely where friction between the heterogeneous fracture surfaces begins. A loading rate of $1.5 \mathrm{~mm} / \mathrm{min}$, or a shear strain of $0.03 / \mathrm{min}$, was used for all tests. On one test, a measurement was taken of the rotation of the loading platen, in order to verify that stresses were as purely shear as possible without rotation. The measurement was taken at the top of the right loading platen (see figure 1). The transverse displacement was measured to be approximately $0.1 \mathrm{~mm}$ just after the ultimate strain was passed, and eventually reached $1 \mathrm{~mm}$ at 0.11 strain. At ultimate, the shear strain was 0.03 , equivalent to about $1.5 \mathrm{~mm}$ of displacement. Since the free length of the loading platen was $300 \mathrm{~mm}$, it suggests the platen rotation was $\arcsin (0.1 \mathrm{~mm} / 300 \mathrm{~mm})=0.02 \mathrm{deg}$.

Distinct diagonal shear cracks formed in the specimens (figure 6) and the material deformed to visible shear strains during the test. The shear modulus was found to be 650 $\mathrm{MPa}$ with a range of plus or minus $40 \mathrm{MPa}$; the shear yield stress $3.3 \pm .3 \mathrm{MPa}$; the yield shear strain $0.007 \pm 0.001$; the shear strength $4.0 \pm 0.4 \mathrm{MPa}$; and the ultimate shear strain $0.026 \pm 0.004$.

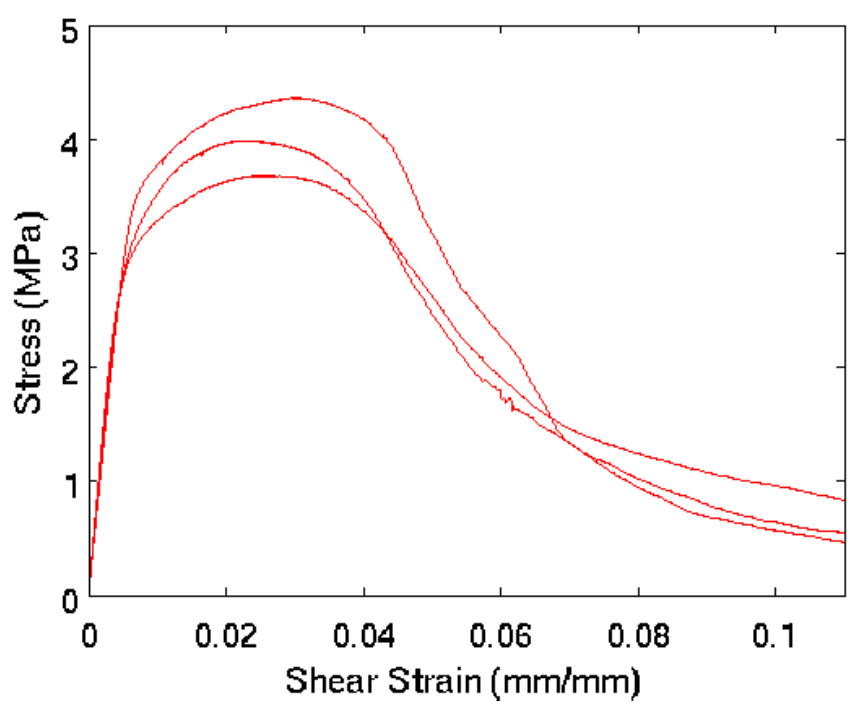




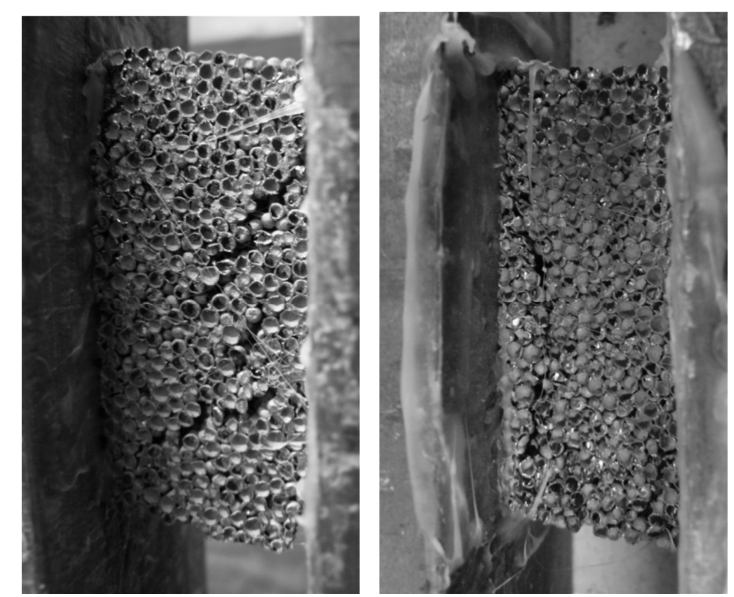

Figure 7: Above: Experimental shear stress/strain curves; Below: Two shear specimens at about 0.08 strain, showing shear cracks.

\subsection{Summary of experimental testing}

The compressive behavior of the HS foam we tested is similar to that reported for other types of metallic foams with two major exceptions. While it exhibits a long phase of compressive ductility between yield and the onset of densification, we observed the presence of a significant hardening modulus that would render a perfectly-plastic assumption difficult to justify. We also observed a very small value for Poisson's ratio in the early stages of deformation followed by a variable Poisson's ratio that peaks at approximately $v=0.30$ near an applied strain of approximately 0.04 . These values of Poisson's ratio differ from those reported for other metal foams, and should be considered carefully when attempting to model the behavior of this HS foam.

Tensile behavior, which is rarely reported for foams, exhibited limited but non-zero ductility, a well-defined yield point, and strength comparable to the yield stress in compression. Investigation of fracture surfaces indicate that macroscopic fracture is caused at the microscale by failure of the hollow sphere shells at the points of connection to neighboring spheres, and the use of thicker spheres has the potential to mitigate this microscale failure mode. 


\section{Continuum constitutive model for steel foams}

The experimental results described in the previous sections reveal several features of the response of the HS steel foam that complicate continuum constitutive modeling. Chief among these are the ability of the material to undergo volume change during inelastic deformation (Poisson's ratio not equal to 0.5 ) and the presence of rapid stiffening of the material after densification initiates at engineering strains around 0.65. These two features mean that standard J-2 plasticity theory cannot be applied to model HS steel foams since the evolution of the yield surface depends not only on deviatoric stress invariant $J_{2}$ but also on the trace of the stress tensor $I_{1}$.

Miller [27], and later Deshpande and Fleck [20] generalized von Mises-Huber plasticity formulation in order to account for the pressure dependence of the yield surface. This formulation is called ' $\mathrm{D}-\mathrm{F}$ ' plasticity throughout this paper. The generalization can be explained by recalling formulae for elastic strain energy of compressible materials [21]:

$$
w_{e}=\frac{1}{2 E} \frac{1}{1+(\alpha / 3)^{2}}\left[\sigma_{e}{ }^{2}+\alpha^{2}{\sigma_{m}}^{2}\right]
$$

$E=$ elastic modulus, and $\alpha=$ compressibility parameter, $\sigma_{e}=$ von Mises effective stress,

$$
\sigma_{e}=\sqrt{3 / 2 \sigma_{i j}^{\prime} \sigma_{i j}^{\prime}}
$$

and, $\sigma_{m}$ hydrostatic pressure or mean stress:

$$
\sigma_{m}=1 / 3 \sigma_{i i}
$$

where the compressibility parameter is a function of Poisson's ratio, $v$

$$
\alpha^{2}=\frac{9}{2} \frac{(1-2 v)}{(1+v)}
$$

Poisson's ratio reflects material compressibility [21]. The compressibility parameter evaluates to $\alpha=0$ for Poisson's ratio of 0.5 , and characterizes an incompressible material. On the other hand, $v=0.0$, and $\alpha=2.12$ indicate high compressibility, and in such case, both pressure, $\sigma_{m}$ and deviatoric stress, $\sigma_{e}$ contribute to the strain energy.

Comparing strain energy at the onset of yield in a uniaxial test, 


$$
w_{u n i}=\frac{\hat{\sigma}^{2}}{2 E}
$$

with the general expression for the elastic energy (equation 3) suggests the following formula for the equivalent yield stress under multi-axial stress state [20,21]:

$$
\hat{\sigma}^{2}=\frac{1}{1+(\alpha / 3)^{2}}\left[\sigma_{e}^{2}+\alpha^{2}{\sigma_{m}}^{2}\right]
$$

It is worth noting that this yield criterion reduces to von Mises formulation, when $\alpha=0$ (and $v=0.5$ ) in the case of plastic incompressibility. Deshpande and Fleck [20] verified their hypothesis by conducting a number of experiments on aluminum foam samples. Their criterion, calibrated against a uniaxial test, agreed well with multi-axial tests.

The key feature of the D-F yield formulation is that it predicts yield under hydrostatic pressure - the 'crushability' that is a feature of all foamed materials. This feature of the yield surface is shown in figure 8, in which the D-F yield surface (the ellipsoid) is superimposed on the cylinder of the classical von Mises theory. The susceptibility of foams to yield under hydrostatic stress appears as the closure of the D-F ellipsoid along the $\sigma_{1}=\sigma_{2}=\sigma_{3}$ direction, where the von Mises cylinder is open in that direction. The degree of crushability is controlled by the parameter $\alpha$, which in turn is determined completely by the value of the plastic Poisson's ratio $v_{p}$ which is 0.5 for solid metals, indicating that the material cannot crush, and is typically below 0.3 for metal foams. The two frames of the figure show the D-F ellipsoid for increasing values of $v_{p}$ illustrating that as $v_{p}$ approaches 0.5 the ellipsoid approaches the classical von Mises cylinder. The figure also shows that the diameter of the D-F ellipsoid exceeds the diameter of the equivalent von Mises cylinder when $v_{p}<0.5$, meaning that a crushable foam may under certain loading scenarios appear stronger than a non-crushable material with the same uniaxial behavior. 

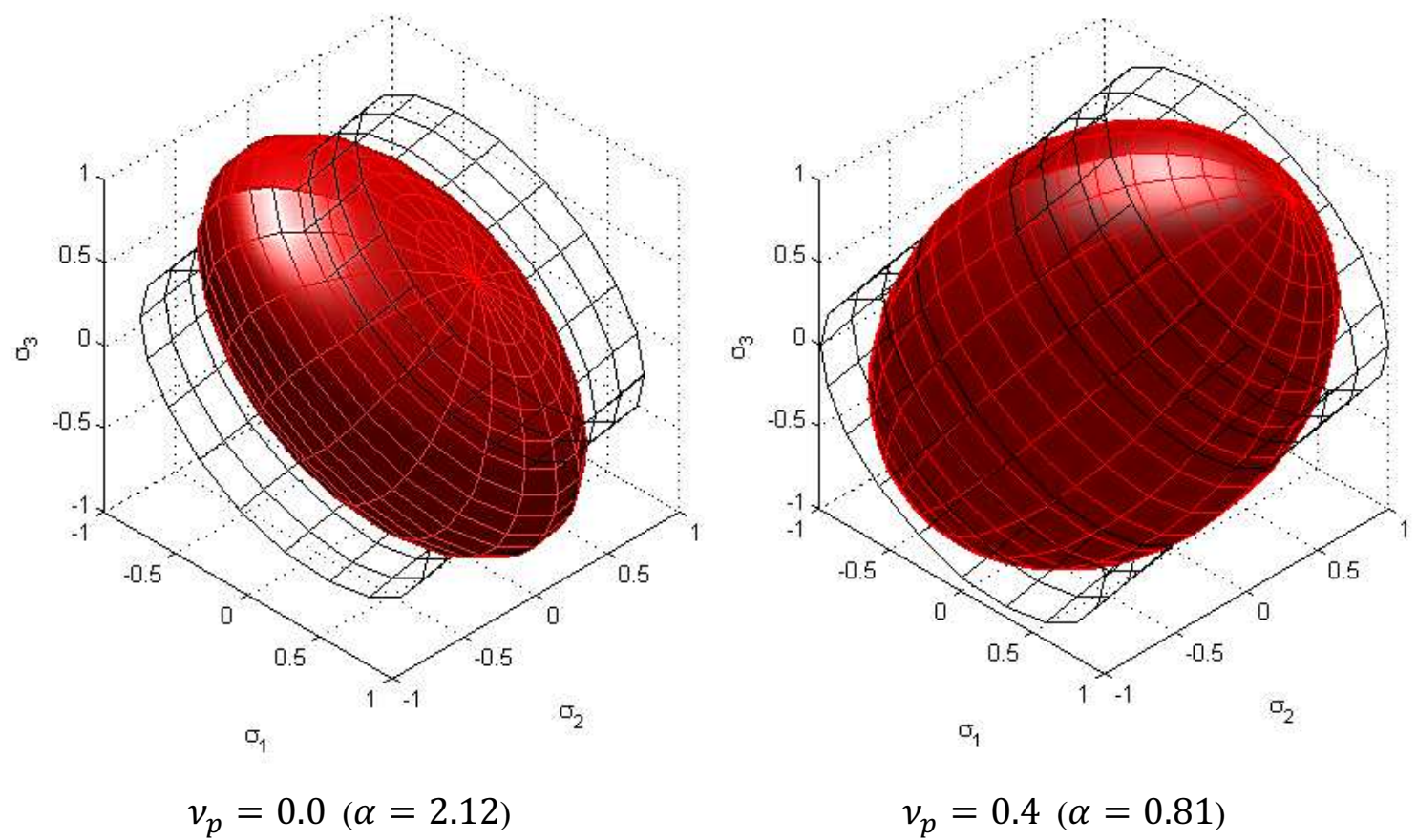

Figure 8: Deshpande-Fleck yield surface (red color). Wire mesh depicts von Mises cylindrical yield surface, calibrated against the same uniaxial stress-strain data. Left: Compressible foam with

Poisson's ratio 0.0. Right: Poisson's ratio approaches 0.5 , and the ellipsoidal yield surface approaches the von Mises cylinder.

To calibrate a D-F plasticity model for a particular material, plastic Poisson's ratio, $v_{p}$ must be measured experimentally, a sensitive task for highly heterogeneous materials such as metal foams. True Poisson's ratio is based on increments of true strains, which can be obtained from previously measured engineering strains:

$$
v_{p, \text { true }}=-\frac{\Delta \varepsilon_{\text {trans,true }}}{\Delta \varepsilon_{\text {axial }, \text { true }}}=-\frac{\Delta \ln \left(1+\varepsilon_{\text {trans,eng }}\right)}{\Delta \ln \left(1+\varepsilon_{\text {axial }, \text { eng }}\right)}
$$

where $\Delta$ indicates strain increments.

Figure 9 shows true Poisson's ratio, $v_{p}$, for the hollow sphere foam described in this paper. The plastic Poisson's ratio in D-F formulation is based on true strains, and not engineering strains. It is important to note that logarithmic conversion of engineering to true strains increases values in compression (for example, $\varepsilon_{\text {eng }}=-0.65 \rightarrow \varepsilon_{\text {true }}=$ -1.05 ), and reduces values in tension (for example, $\varepsilon_{\text {eng }}=0.05 \rightarrow \varepsilon_{\text {true }}=0.49$ ). Since transverse tensile strains are reduced during the conversion, and axial compressive strains are increased by the conversion, true Poisson's ratios in figure 9 have smaller 
numeric values than engineering Poisson's ratios in figure 5. The D-F model allows for a single value of $v_{p}$ and the best fit value $v_{p}=0.12$ is shown in a bold solid line superimposed on the experimental results. Classical J2 plasticity would incorrectly predict Poisson's ratio of 0.5 .

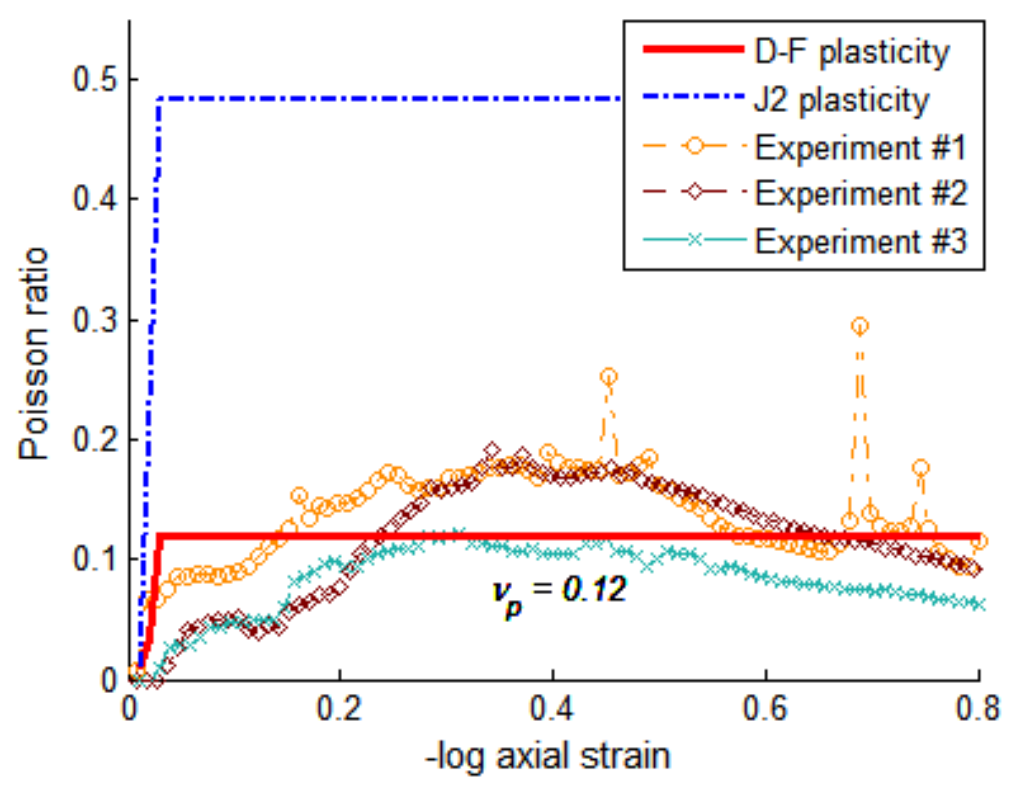

Figure 9: True plastic Poisson's ratio

D-F plasticity is available in LS-DYNA [28], and the implemented model was used in this study. The uniaxial hardening in LS-DYNA is approximated by the analytical expression [21] to allow robust numerical implementation:

$$
\hat{\sigma}_{y}=\sigma_{p}+\gamma \frac{\hat{\varepsilon}}{\epsilon_{D}}+\alpha_{2} \ln \left(\frac{1}{1-\left(\frac{\hat{\varepsilon}}{\varepsilon_{D}}\right)^{\beta}}\right)
$$

where $\hat{\varepsilon}=$ effective plastic strain, $\epsilon_{D}=-\ln (\rho)$, where $\rho=$ foam relative density, and $\sigma_{p}, \gamma, \alpha_{2}, \beta$ are obtained by the curve-fit to the experimental curve (figure 10).

The model is calibrated against true strains and true Cauchy stress. Although, true strains can be obtained with classical conversion $\varepsilon_{\text {true }}=\ln \left(1+\varepsilon_{\text {eng }}\right)$, true stress is a function of the actual cross-section. Thus, measurements of transverse strains during the axial 
experiment are required, not only for determination of Poisson's ratio, but also for calculation of true stresses from the applied force history.

$$
\sigma_{\text {true }}=\frac{P}{b^{2}}=\frac{P}{b_{0}{ }^{2}\left(1+\varepsilon_{\text {trans }, \text { eng }}\right)^{2}}=\sigma_{\text {eng }}\left(1+\varepsilon_{\text {trans }, \text { eng }}\right)^{-2}
$$

where $\sigma=$ axial stress, $P=$ applied force, $b=$ current transverse dimension, $b_{0}=$ original transverse dimension, $\varepsilon_{\text {trans,eng }}=$ engineering transverse strain.

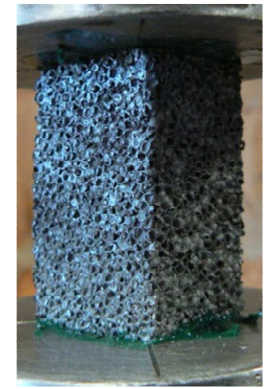

$\epsilon_{\text {eng }}=0.10$

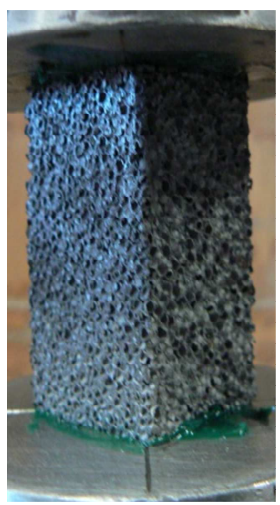

initial
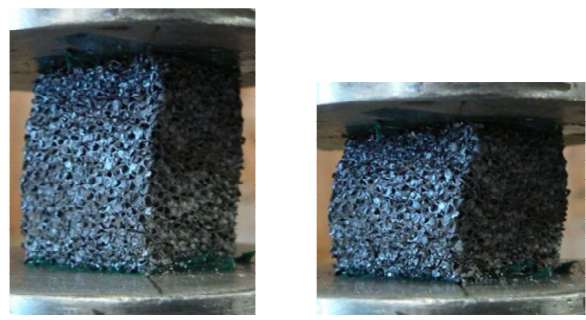

$\epsilon_{\text {eng }}=0.35$

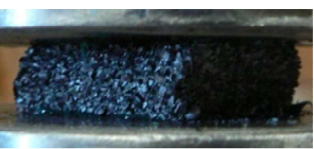

final

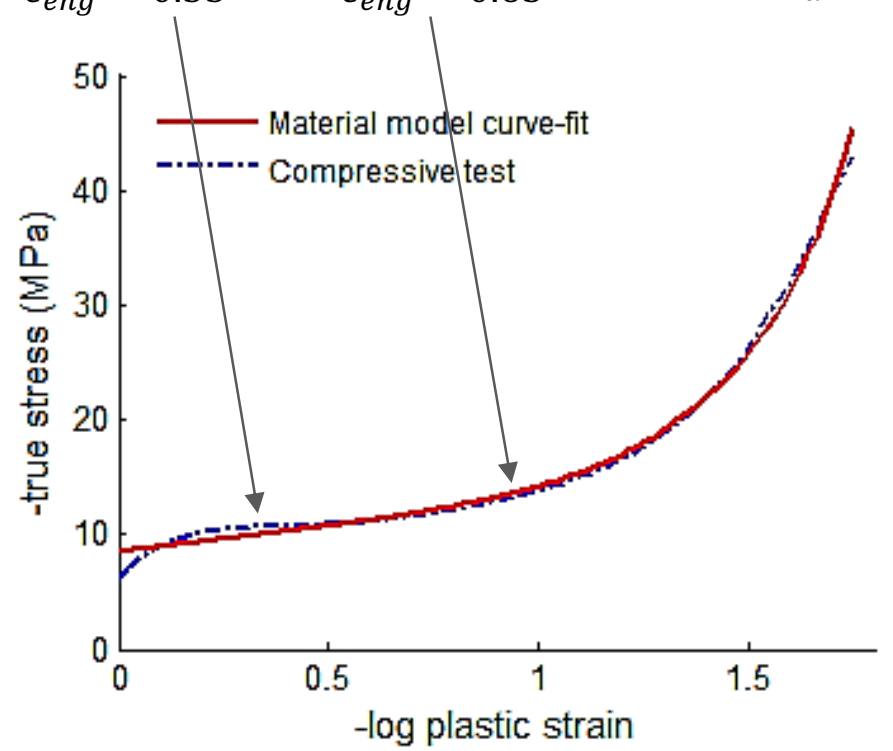

Figure 10: Curve-fit of analytical hardening curve to experimental measurements

The classical formula for conversion of engineering to true stress

$$
\sigma_{\text {true }}=\sigma_{\text {eng }}\left(1+\varepsilon_{\text {axial }, e n g}\right)
$$

is based on assumption of incompressibility, and it is not applicable to compressible materials. The general conversion formula, based on the assumption of constant true plastic Poisson's ratio, is: 


$$
\sigma_{\text {true }}=\sigma_{\text {eng }}\left(1+\varepsilon_{\text {axial }, \text { eng }}\right)^{2 v_{\text {p.log }}}
$$

where $v_{p . \log }=\log$ plastic Poisson's ratio.

Since plastic Poisson's ratio varies with applied strains, direct measurements of the actual cross-sectional area (changes in transverse dimensions of a specimen) are preferred.

Table 2 summarizes the mechanical properties measured during this series of experiments, and includes parameters corresponding to the Deshpande-Fleck material model. This table of material properties should provide sufficient information for an analyst or designer interested in developing structural applications of HS steel foams to perform finite element simulations of component response.

Table 2: summary of HS steel foam material properties.

Material properties:

\begin{tabular}{ll}
\hline Relative density, $\rho$ & 0.145 \\
Elastic modulus & $3150 \mathrm{MPa}$ \\
Poisson's ratio (elastic) & 0.05 \\
\hline Yield surface: & \\
\hline Curve-fitted plateau stress, $\sigma_{p}$ & $7.4 \mathrm{MPa}$ \\
Shape parameter, $\alpha$ & 1.75 \\
\hline Hardening: & \\
\hline Curve-fitted, $\gamma$ & 10.9 \\
Curve-fitted, $\alpha_{2}$ & 33.2 \\
Curve-fitted, $\beta$ & 5.5 \\
\hline Failure: & \\
\hline Tensile fracture strain & 0.02
\end{tabular}

\section{Constitutive model validation}

\subsection{Tensile and compressive verification simulations}

In order to assess the ability of the D-F plasticity model to reproduce the behavior of hollow sphere steel foam accurately, finite element models of the tensile, compressive, 
and shear test coupons were developed and the simulated response is compared to the experimental results.

Reyes et al. [21] and Hansen et al. [22] enhanced D-F plasticity with the tensile fracture criteria based on the maximum principal stress and this enhanced model is implemented in LS-DYNA [28], the software used in this study. As of version 9.71 release 5.1.1, LSDYNA requires tensile fracture strain for the calibration of this element erosion criterion. Fracture strain of 0.02 , a lower bound from uniaxial tensile experiments, was selected as input to LS-DYNA.

The yield stress, Young's modulus, and fracture strain were modeled as spatially varying random field with coefficient of variation equal to $20 \%$, and a spatially isotropic correlation length of $2 \mathrm{~mm}$ corresponding to roughly one sphere diameter. Since yield plateau is correlated with the fracture strain (see figure 5); yield stress, Young's modulus and the failure strain were assumed to be perfectly correlated. In simple terms, simulated elements with lower yield stress also exhibited reduced material stiffness and fracture strain, and vice versa.

The numerical simulations, which produced fracture at varying locations in the coupon, are qualitatively consistent with the experimental tests elaborated earlier in this paper. Specifically, the introduction of spatial variation into the material property field successfully drives the site of fracture initiation away from the coupon bevel, consistent with experimental results (figure 11). A deterministic model failed at the bevel due to a stress concentration at that location ( $5 \%$ higher stress then in the reduced section).
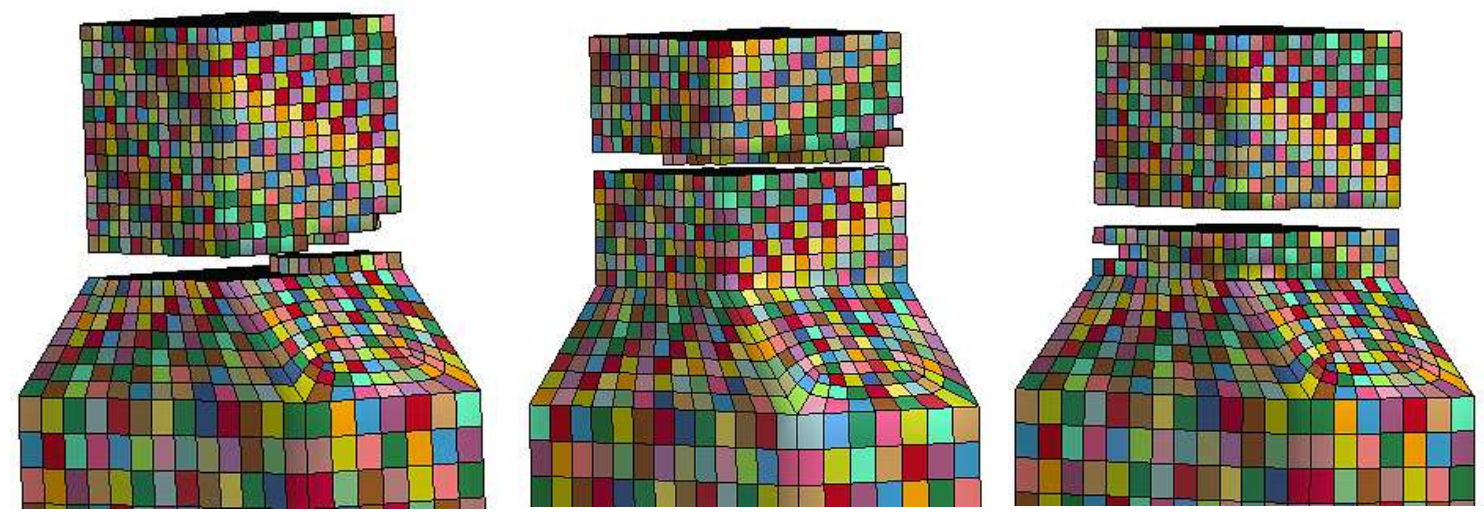

Figure 11: Sample numerical realizations of tensile fracture. 
Note: colors represent model components with randomly assigned, unique material properties.

Colors are cycled (due to limited number of available colors), thus two identically colored elements do not have the same material properties.

Compressive simulations of the prismatic specimen used in the experiments produced non-uniform deformation fields and uneven external surfaces (figure 12) when the yield stress and elastic modulus were treated as perfectly correlated random fields as they were in the tension simulations. As expected, the compressive behavior was less sensitive to heterogeneity of the material property field. When the material properties were treated as spatially homogeneous (i.e. non-random field) the simulated response also reasonably approximated that observed in the experiments (figure 13).
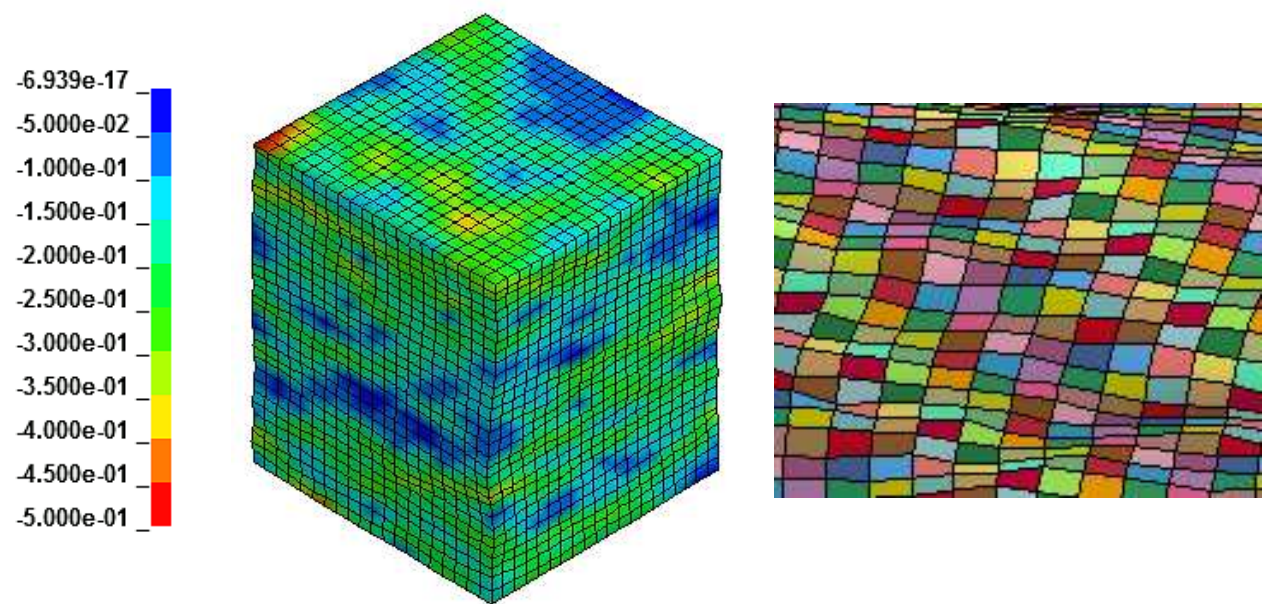

Figure 12: Numerical realization of the compressive test with axial strains plotted. Note nonuniform axial deformations.

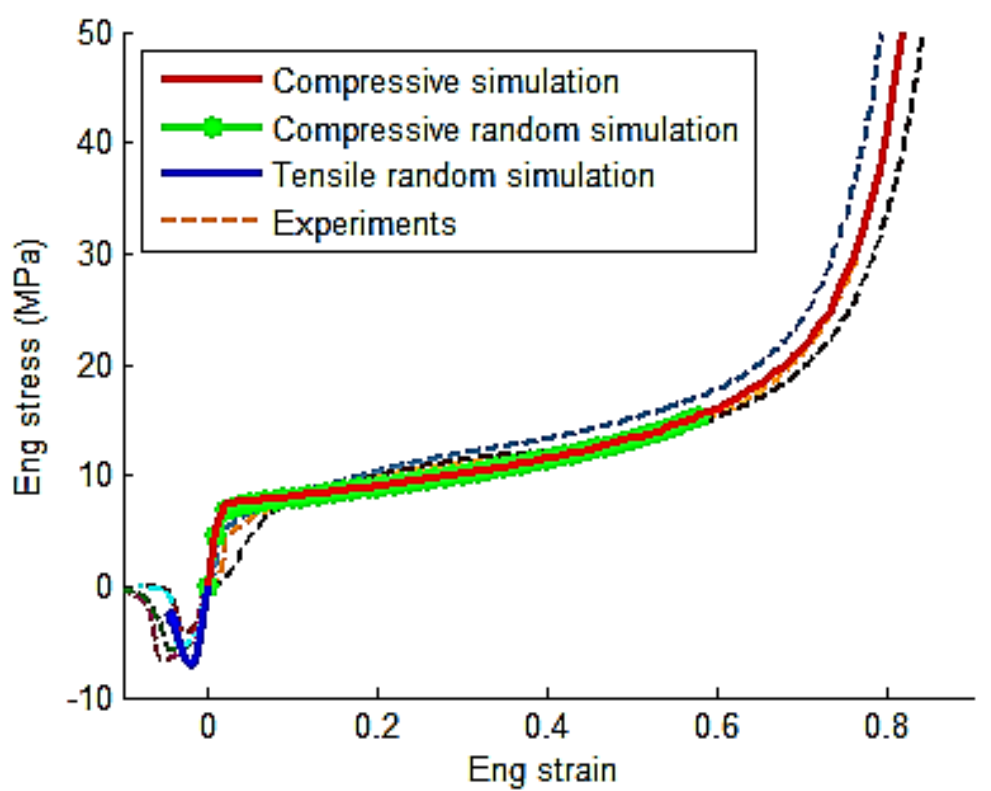


Figure13: Calibration (compression) and verification (tension) of numerical model against experiments

\subsection{Shear validation simulations}

The D-F yield criterion predicts yield in shear at a stress level somewhat higher than predicted by von Mises plasticity, but still lower than the uniaxial yield stress. In D-F plasticity the yield stress in shear $\sigma_{s y}$ is given by

$$
\sigma_{s y}=\sqrt{\frac{1+\frac{\alpha^{2}}{3}}{3}} \sigma_{u y}=\sqrt{\frac{1}{2\left(1+v_{p}\right)}} \sigma_{u y} \geq \sigma_{J 2}=\frac{1}{\sqrt{3}} \sigma_{u y}=0.58 \sigma_{u y}
$$

in which $\sigma_{u y}$ is uniaxial yield stress and $\sigma_{j 2}$ is the von Mises yield stress in shear. The von Mises formulation predicts yield shear stress of $\sigma_{J 2}=0.58 \cdot 4 \mathrm{MPa}=2.3 \mathrm{MPa}$. For the hollow sphere foam studied here, with $v_{p}=0.12$, D-F plasticity predicts $\sigma_{s y}=$ $0.67 \sigma_{u y}=0.67 \cdot 4 \mathrm{MPa}=2.7 \mathrm{MPa}$. Average experimental shear yield stress was 3.3 $\mathrm{MPa}$. Thus, D-F plasticity provided more accurate prediction of the tested values than the classical $\mathrm{J} 2$ plasticity.

Shear deformation produces no volumetric strain, and therefore element deletion criteria that rely on volumetric strain cannot be used to simulate shear failure. Reyes et al. [21] proposed the use of the maximum principal stress in place of volumetric strain as an element deletion criterion. As implemented in LS-DYNA version 9.71 release 5.1.1, the maximum principal stress criterion does not produce shear fracture patterns that resemble those observed in experiments (Compare figures 14c and 14a), although it does generate good predictions of the strength and post-peak response. Therefore, a new element deletion criterion based on equivalence of the maximum principal strain to the tensile fracture strain $\left(\varepsilon_{\max }=0.02\right)$ was proposed, and it was implemented in LS-DYNA [28] with *MAT_ADD_EROSION keyword. Simulations using this criterion generated fracture patterns that are reasonably similar to those observed in experiments. Simulated cracks propagated along one of the fixture plates in approx. $70 \%$ of numerical realizations, and in the remaining $30 \%$ of realizations the erosion path turned toward the centerline (compare figures 14b and 14a). This is in agreement with experiments, since two of the 
tested specimens developed a crack along one of the loading platens, and one specimen exhibited diagonal cracks (see figure 7).
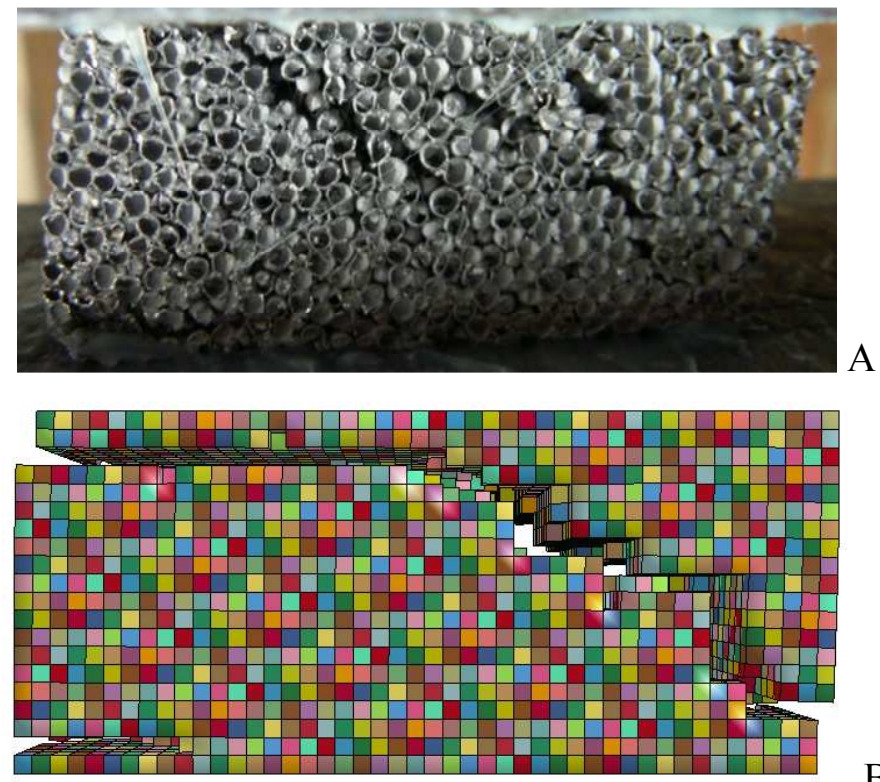

B

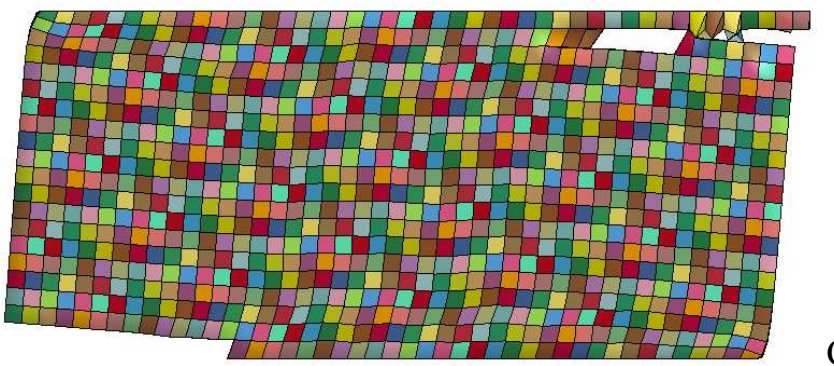

$\mathrm{C}$

Figure 14: Numerical simulation of the shear tests: A) Experiment, B) Postulated material erosion based on the maximum principal strain at failure, C) Element deletion based on the maximum principal stress.

Principal strain based approach provided good predictions of the strength and crack patterns, but did not capture the post-peak residual capacity of the specimen (figure 15). The most likely explanation for this discrepancy in post-peak behavior is that the element deletion algorithm leaves a gap between the fracture surfaces, whereas in the physical experiments contact of the rough fracture surfaces occurs and allows the material to maintain significant load carrying capacity. The fracture paths in the experimental sample are also more tortuous than those produced by the simulations, further enhancing the potential load transfer across the crack. Of the two element deletion criteria investigated, both predict the strength well, with the stress-based criterion overestimating ductility and 
the strain based criterion underestimating ductility, and thus providing a more conservative estimate of failure. Further investigation of the appropriate element deletion criterion would be an interesting pursuit, but lies beyond the scope of this paper.

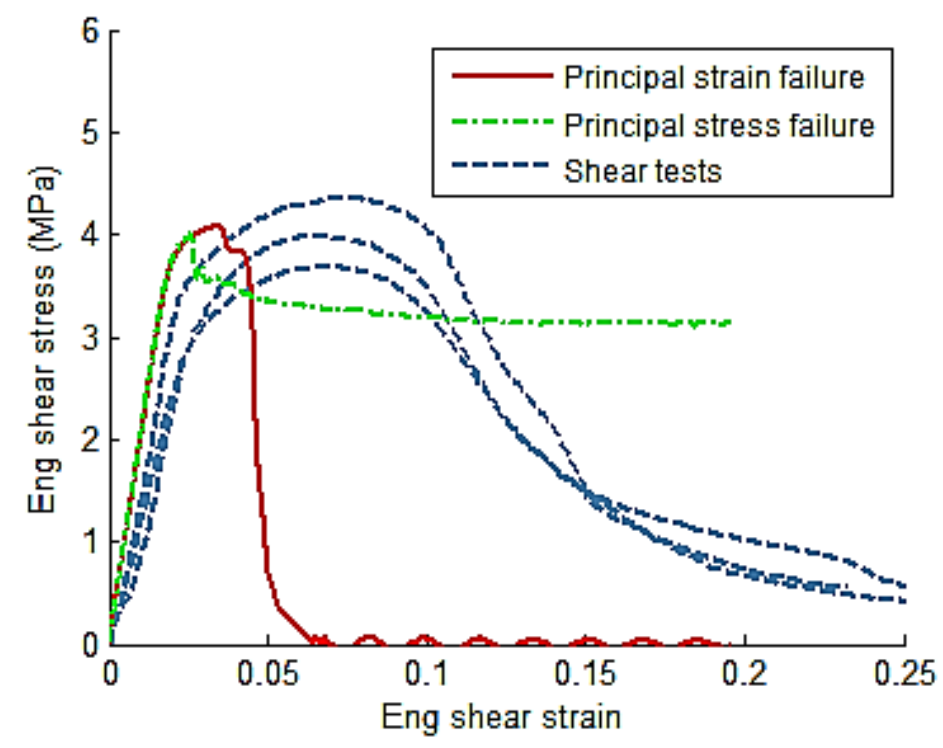

Figure 15: Comparison of shear simulations with experiments

\section{Importance of Poisson's ratio}

Having shown that D-F plasticity can provide a good approximation to the physical behavior of hollow sphere steel foam in the three canonical loading modes (tension, compression and shear), the D-F plasticity formulation is now used to explore the effect of Poisson's ratio on response of steel foam components under multi-axial stress states.

\subsection{Effect of Confinement on Compressive Behavior}

A finite element model of a prismatic block of HS steel foam was developed in LSDYNA and material properties calibrated to the experimental tests were assigned to the individual elements in the form of a spatially varying random field as described previously. This model was then exercised using boundary conditions corresponding to laterally confined compression $\left(\varepsilon_{1}<0, \varepsilon_{2}=\varepsilon_{3}=0\right)$, and triaxial compression $\left(\varepsilon_{1}=\varepsilon_{2}=\varepsilon_{3}\right.$ $<0)$. Such conditions regularly occur in structural applications and the behavior of HS steel foam under such conditions must be understood before the material can be widely 
deployed. While a formal experimental investigation is needed, these numerical experiments provide an indication of the types of behavior to be expected.

The most interesting aspects of our particular HS steel foam $\left(v_{e}=0.01, v_{p}=0.12\right)$ behavior under multiaxial loading is that confinement does not increase the magnitude of surface traction (termed further as apparent yield stress) needed to yield the confined specimen. Also, under triaxial loading the apparent yield stress is actually lower than the apparent yield stress in uniaxial compression (figure 16a), although the material does eventually stiffen and harden under triaxial loading. This feature of the response can also be seen in figure 8 left in which the diameter of the D-F ellipsoid along the $\sigma_{1}=\sigma_{2}=\sigma_{3}$ axis is smaller than the radius of the von Mises cylinder.

It should be noted that other types of foams with Poisson's ratio greater than 0.33 may exhibit the opposite behavior, because Poisson's ratio affects the apparent yield stress of the confined HS foam. The applied surface traction to yield the confined specimen can be analytically expressed by deriving the stress state corresponding to non-zero axial strain, and zero transverse strains, and later substituting these stress components into the D-F yield criterion, to obtain:

$$
\sigma_{\text {confy }}=\sqrt{\frac{1+\frac{1}{2} \frac{\left(1-2 v_{p}\right)}{\left(1+v_{p}\right)}}{\frac{1}{\left(1+v_{e}\right)^{2}}+\frac{9}{2} \frac{\left(1-2 v_{p}\right)}{\left(1+v_{p}\right)}\left(\frac{1}{3\left(1-v_{e}\right)}\right)^{2}}} \sigma_{\text {uniaxy }}
$$

which depends on the elastic and plastic Poisson's ratios $v_{e}$ and $v_{p}$ (figure 16b). The figure 16b shows that when the elastic Poisson's ratio is very small the confined yield stress is essentially equal to the unconfined yield stress, as is the case in the current HS steel foam with $v_{e}=0.01$.

Only when $v_{e}$ becomes substantially larger than zero, confinement creates transverse stress resulting in triaxial pressure. The triaxial pressure lowers the apparent yield stress for foams with $v_{p}<0.33$, and increases the apparent yield stress for foams with $v_{p}>$ 0.33. It is important to note, first, that Deshpande and Fleck [20] experimentally probed the yield surface of foams, and that therefore this result for the HS foam can be 
considered instructive even though multiaxial physical tests have not been performed, and second, that by tuning $v_{e}$ and $v_{p}$, it is in fact possible to generate a D-F material with a higher confined than unconfined yield stress. Such tuning of the Poisson's ratio is possible through control of the microstructure and porosity.
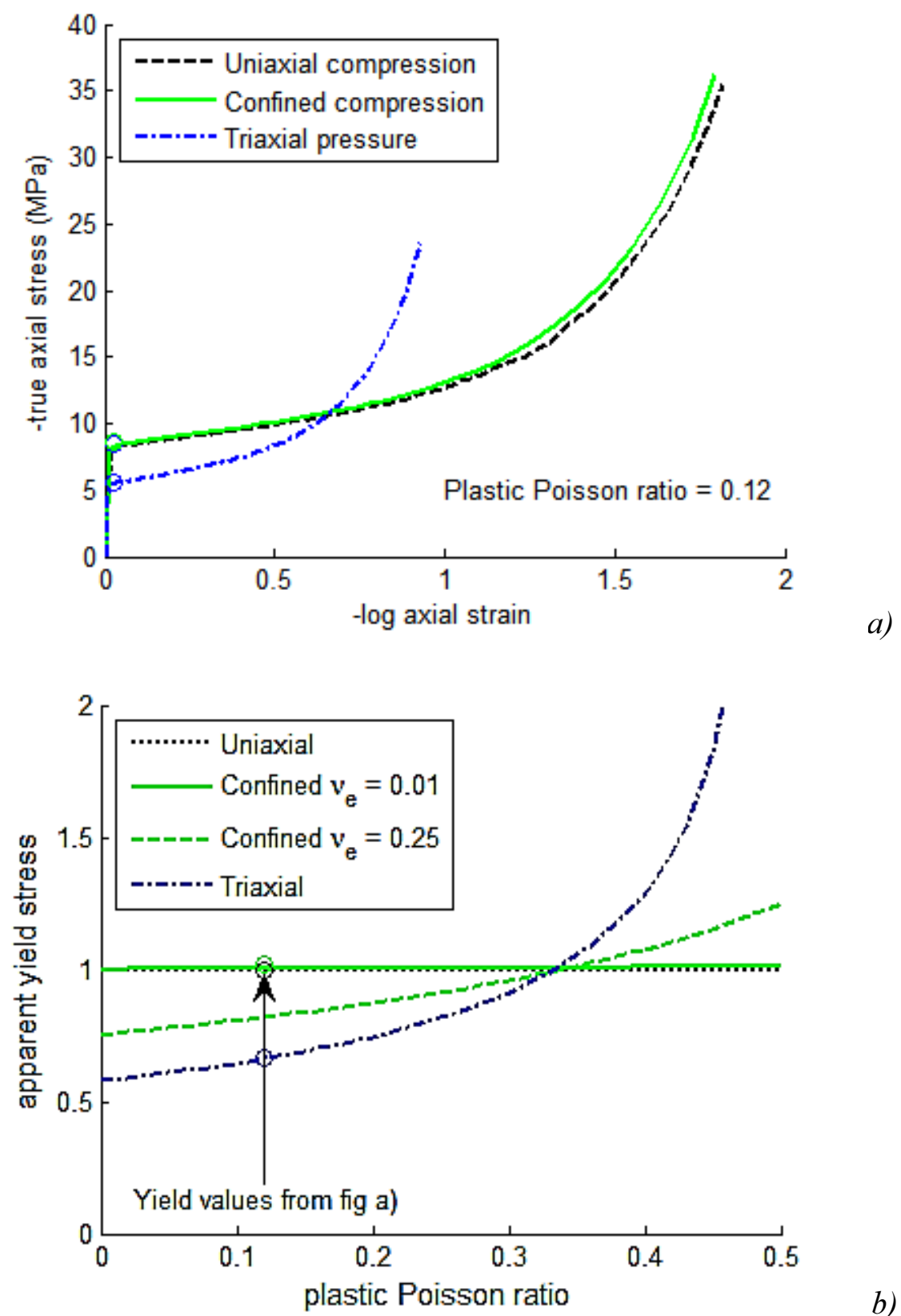

b)

Figure 16: a) Stress-strain histories from simulations of our HS foam, characterized with elastic $v_{e}=0.01$ and plastic Poisson's ratio $v_{p}=0.12$, b) Aggregated apparent yield values, normalized by uniaxial yield, from a parametric sweep of plastic Poisson's ratios $v_{p}=0.0 \sim 0.5$. Since confinement stress depends on elastic Poisson's ratio, two curves for elastic Poisson's ratio 
of 0.01 and 0.25 are plotted. Note that onsets of yield from figure a) correspond to 3 points in figure $b$ ).

The reduction in triaxial yield stress of our HS foam also arises directly from the D-F yield criterion (with deviatoric term $\sigma_{e}=0$ under triaxial pressure), in which the triaxial yield stress, $\sigma_{m y}$, expressed as a function of uniaxial yield stress, is

$$
\sigma_{m y}=\frac{\sqrt{1+\frac{\alpha^{2}}{3}}}{\alpha} \sigma_{y}=\sqrt{\frac{1}{3-6 v_{p}}} \sigma_{y}
$$

where $\alpha=\sqrt{\frac{9}{2} \frac{\left(1-2 v_{p}\right)}{\left(1+v_{p}\right)}}=$ yield surface parameter, $v_{p}=$ plastic Poisson's ratio Triaxial yield stress, $\sigma_{m y}$ becomes infinite if $v_{p}$ approaches 0.5 (incompressible plasticity), and gives $\sigma_{m y}<\sigma_{y}$ when $v_{p}<0.33$ as is the case for our HS steel foam with $v_{p}$ $=0.12$ (see figure $16 \mathrm{~b})$.

\subsection{Foam compaction}

Another key property of foams is the compaction strain - the compressive strain at which foam porosity is zero, and all pores are completely closed. Compaction strain should not be confused with the densification strain - the compressive strain at which the foam begins to rapidly stiffen and harden due to collapse of the cells and widespread contact between the cell walls. Compaction strain is an important design parameter because it effectively defines the amount of compressive deformation that can be accommodated by a foam without the transfer of stresses at high levels of the base foam material occurring. The D-F plasticity model defines a compaction strain that is dependent on the plastic Poisson's ratio and relative density $\rho$. The logarithmic compaction strain in unconfined axial compression is [21]

$$
\varepsilon_{D}=-\frac{9+\alpha^{2}}{3 \alpha^{2}} \ln (\rho)=-\frac{1}{1-2 v_{p}} \ln (\rho)
$$

which can be converted to an engineering value by 


$$
\varepsilon_{\text {Deng }}=e^{\varepsilon_{\mathrm{D}}}-1=\rho^{\frac{1}{1-2 v_{p}}}-1
$$

It is self-evident that the compaction strain will be smaller for foams with higher relative density (and inherent lower porosity). Furthermore, the compaction strain becomes larger (see figure 17) as the incompressibility increases, characterized by the larger Poisson's ratio. This counterintuitive behavior stems from the fact that, under uniaxial compression, higher incompressibility results in transverse creeping of the material, and thus increases uniaxial strain needed to close all the pores. This is another material response domain in which the crushability of the foam, as characterized by the plastic Poisson's ratio, can dramatically affect their behavior.

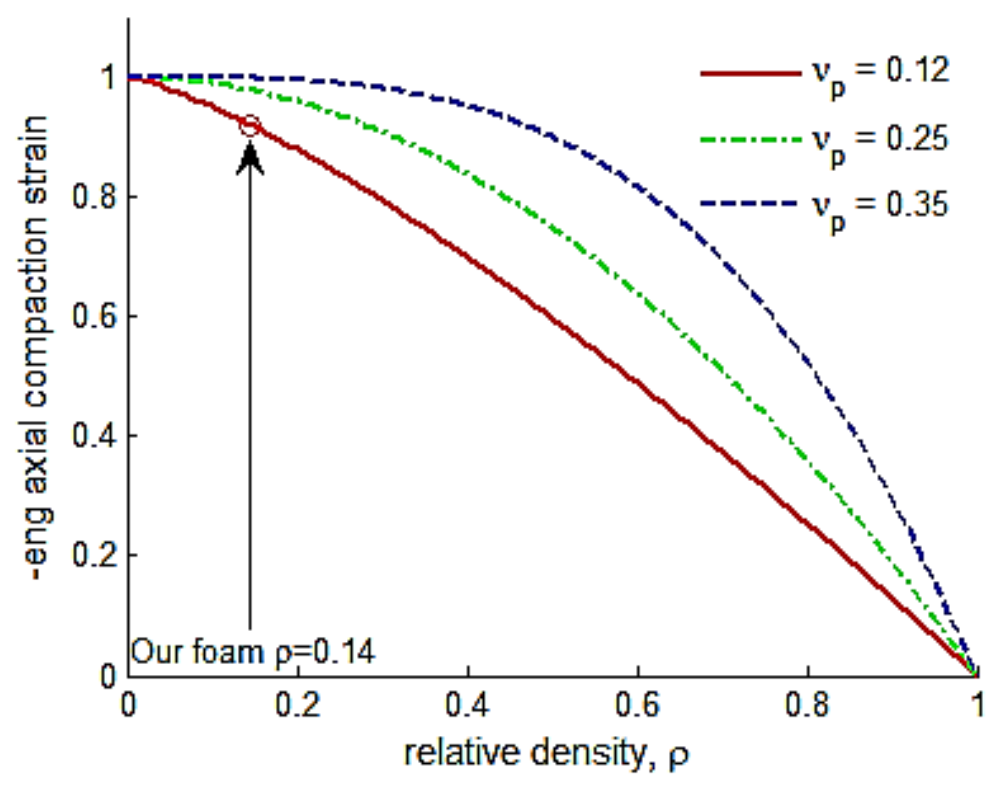

Figure 17: Effect of relative density and plastic Poisson's ratio on uniaxial compaction strain

\subsection{Discussion of simulations and modeling approach}

D-F plasticity formulation, which accounts for material compressibility under triaxial pressure, offers a more physical material representation than classical J2 plasticity, which assumes material incompressibility. Our experimental shear results confirmed the efficacy of D-F plasticity for modeling of hollow sphere foams. It is also important to note that D-F plasticity operates on logarithmic strains and true stresses, which require 
measurements of the actual cross-sectional dimensions during uniaxial experiments. Such measurements are also need for quantification of Poisson's ratio, which determines the shape of the D-F yield surface.

Inclusion of random variability of mechanical properties in hollow sphere foams enhances modeling of fracture patterns, and realism of failure simulations. Principal strain based formulation was more accurate in reproducing experimental shear fracture patterns than principal stress based approach.

Foam Poisson's ratio is indispensable for quantification of compressibility, but is rarely reported in experimental papers, and its theoretical estimate [2] is thought to be 0.3 . However, our experimental tests indicated that true plastic Poisson's ratio can be as low as 0.12 , and its value is a critical indicator of material compressibility. Plastic Poisson's ratio impacts the shape of the D-F yield surface, changes resistance of confined components to axial loading, and controls the value of the compaction strain. Plastic Poisson's ratio is a fundamental characteristic of compressible foams, and should be routinely reported in experimental papers.

\section{Conclusions}

This paper characterizes mechanical properties of steel hollow sphere (SHS) foam, and applies calibrated Deshpande-Fleck plasticity to mechanical simulations of steel foam components. The experimental characterization of the hollow sphere foam encompassed compressive yield stress and densification strain, compressive plastic Poisson's ratio, compressive unloading modulus, as well as tensile elastic modulus, tensile unloading modulus, tensile yield stress, tensile fracture strain, and shears yield stress and shear fracture strain. A definition of densification strain was expressed in mathematical terms based on rigorous regressions. Novel testing approaches for shear and tensile properties of HS steel foams were also proposed.

HS steel foam is a compressible material, with exceptional deformation capabilities under compressive loads (up to 0.9 engineering strain). While it exhibits a long phase of compressive ductility between yield and the onset of densification, we observed the 
presence of a significant hardening modulus that would render a perfectly-plastic assumption difficult to justify. We also observed an average value of true Poisson's ratio to be approximately 0.12 ; and this value of Poisson's ratio differs from those reported for other metal foams [2].

Tensile behavior exhibited limited but non-zero ductility, a well-defined yield point, and strength comparable to the yield stress in compression. Macroscopic fracture was caused by failure of the hollow sphere shells at the connections to neighboring spheres, and the use of thicker spheres may mitigate this failure mode. Shear behavior exhibited limited ductility, reaching ultimate strength at engineering strains between 0.05 and 0.10 .

D-F plasticity [20] is adequate to model plastic behavior of HS foams because it accounts for foam compressibility. D-F provided more accurate prediction of the experimental shear yield stress than classical von Mises plasticity. However, D-F plasticity needs to be supplemented with a suitable fracture criterion in order to account for asymmetry between compressive and tensile fracture, e.g. based on the maximum principal strain. Random distributions of material properties within each component enhanced the realism of the tensile and shear simulations.

Poisson's ratio provides invaluable information about the foam compressibility, which affects the resistance in the case of confined boundaries, and under multi-axial loads. Plastic Poisson's ratio can be obtained from a uniaxial experiment, circumventing the need for specialized multi-axial testing equipment. Since transverse dimensions need to be measured during a uniaxial test in order to calculate true stresses, determination of plastic Poisson's ratio requires only manipulation of the collected data.

Future work is needed on tensile and shear testing standards for metallic foams. Also, evolution of plastic hardening and especially fracture under multi-axial, complex load paths needs further investigation. Random spatial distributions of material properties may also warrant further studies due to their potential impact on reliability of foamed 
components. This work is part of a larger effort to help develop steel foam as a material with relevance to civil engineering applications.

\section{Acknowledgements}

This work was funded by the United States National Science Foundation under grants CMMI- 1000334, 1000167, 0970059. Drs. Hartmut Goehler and Guenter Stephani of the Fraunhofer Institute in Dresden were instrumental in making the hollow sphere foam samples available for testing. Drs. Hartmut Goehler and Guenter Stephani support is hugely appreciated. This work used the Extreme Science and Engineering Discovery Environment (XSEDE), which is supported by National Science Foundation grant number OCI-1053575.

\section{References}

[1] Smith BH, Szyniszewski S, Hajjar JF, Schafer BW, Arwade SR. Steel foam for structures: A review of applications, manufacturing and material properties. Journal of Constructional Steel Research 2012;71:1-10.

[2] Ashby M. Metal foams : a design guide. Boston: Butterworth-Heinemann; 2000.

[3] Mutlu I, Oktay E. Mechanical properties of sinter-hardened $\mathrm{Cr}-\mathrm{Si}-\mathrm{Ni}-\mathrm{Mo}$ based steel foam. Materials \& Design 2013;44:274-82.

[4] Hsu H-C, Wu S-C, Hsu S-K, Tsai M-S, Chang T-Y, Ho W-F. Processing and mechanical properties of porous Ti-7.5Mo alloy. Materials \& Design n.d.

[5] Gibson LJ, Ashby MF, Ashby M. Cellular Solids: Structure and Properties. 2nd ed. Cambridge University Press; 1999.

[6] Bažant Z, Zhou Y. Why Did the World Trade Center Collapse?-Simple Analysis. Journal of Engineering Mechanics 2002;128:2-6.

[7] Szyniszewski S, Krauthammer T. Energy flow in progressive collapse of steel framed buildings. Engineering Structures 2012;42:142-53.

[8] Peroni L, Scapin M, Avalle M, Weise J, Lehmhus D. Dynamic mechanical behavior of syntactic iron foams with glass microspheres. Materials Science and Engineering: A 2012;552:364-75.

[9] Moradi M, Arwade SR, Schafer BW. Computational evaluation of limit states of thin-walled channels made from steel foam. Thin-Walled Structures 2013;62:20614.

[10] Szyniszewski S, Smith BH, Hajjar JF, Arwade SR, Schafer BW. Local buckling strength of steel foam sandwich panels. Thin-Walled Structures 2012;59:11-9.

[11] Banhart J, Seeliger H-W. Aluminium Foam Sandwich Panels: Manufacture, Metallurgy and Applications. Adv Eng Mater 2008;10:793-802.

[12] Hipke T. Personal Communication 2011.

[13] Lefebvre L -P, Banhart J, Dunand DC. Porous Metals and Metallic Foams: Current Status and Recent Developments. Advanced Engineering Materials 2008;10:775-87. 
[14] Fiedler T, Belova IV, Murch GE. Critical analysis of the experimental determination of the thermal resistance of metal foams. International Journal of Heat and Mass Transfer n.d.

[15] Neugebauer R, Hipke T. Machine Tools With Metal Foams. Advanced Engineering Materials 2006;8:858-63.

[16] Han F, Seiffert G, Zhao Y, Gibbs B. Acoustic absorption behaviour of an opencelled aluminium foam. J Phys D: Appl Phys 2003;36:294.

[17] Lu TJ, Chen C. Thermal transport and fire retardance properties of cellular aluminium alloys. Acta Materialia 1999;47:1469-85.

[18] Losito O, Barletta D, Dimiccoli V. A wide-frequency model of metal foam for shielding applications. IEEE Transactions on Electromagnetic Compatibility 2010;52:75-81.

[19] Xu S, Bourham M, Rabiei A. A novel ultra-light structure for radiation shielding. Materials and Design 2010;31:2140-6.

[20] Deshpande VS, Fleck NA. Isotropic constitutive models for metallic foams. Journal of the Mechanics and Physics of Solids 2000;48:1253-83.

[21] Reyes A, Hopperstad OS, Berstad T, Hanssen AG, Langseth M. Constitutive modeling of aluminum foam including fracture and statistical variation of density. European Journal of Mechanics - A/Solids 2003;22:815-35.

[22] Hanssen AG, Hopperstad OS, Langseth M, Ilstad H. Validation of constitutive models applicable to aluminium foams. International Journal of Mechanical Sciences 2002;44:359-406.

[23] Technical Committee ISO/TC 164. Compression test for porous and cellular metals - ISO 133142011.

[24] E28 Committee. Test Methods for Tension Testing of Metallic Materials. ASTM International; 2011.

[25] Technical Committee ISO/TC 61, Plastics, Subcommittee SC 10, Cellular plastics. Rigid cellular plastics. Determination of shear strength - ISO 1922:2012 2012.

[26] Friedl O, Motz C, Peterlik H, Puchegger S, Reger N, Pippan R. Experimental Investigation of Mechanical Properties of Metallic Hollow Sphere Structures. Metallurgical and Materials Transactions B 2008;39:135-46.

[27] Miller RE. A continuum plasticity model for the constitutive and indentation behaviour of foamed metals. International Journal of Mechanical Sciences 2000;42:729-54.

[28] Hallquist J. LS-DYNA theory manual. Livermore, California: Lawrence Software Technology Corporation; 2006. 